\title{
Vaccins contre le SIDA : espoirs et réalités
}

Le développement d'un vaccin actif contre le SIDA serait probablement nécessaire à l'arrêt de l'épidémie actuelle. Les premiers résultats soulignent les difficultés de l'entreprise et le chemin restant à parcourir: les protéines virales ne sont pas toutes correctement antigéniques, les anticorps développés pourraient être parfois facilitants plus que neutralisants, la variabilité génétique du virus HIV l'amène à échapper à la réponse immune, l'immunité cellulaire est probablement indispensable à une bonne protection... mais peut-être insuffisante et peut même jouer un rôle dans certains signes cliniques de la maladie.

\section{Marc Girard}

\section{ADRESSE}

M. Girard: professeur, directeur scientifique de Pasteur-Vaccins, chef de l'unité de virologie moléculaire. Institut Pasteur, 28, rue du Doc-

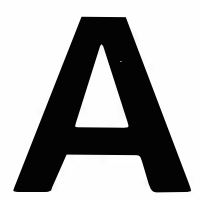

quand un vaccin contre le SIDA ? Cette question se pose avec d'autant plus d'angoisse que la pandémie du SIDA poursuit sa progression sans fléchir et qu'aucune solution satisfaisante ne semble apparaître à l'horizon en matière de chimiothérapie antivirale. Un vaccin efficace serait la meilleure arme contre la maladie. Mais le virus HIV (human immunodeficiency virus) élude pour l'instant les efforts des chercheurs et il est fort à craindre qu'on ne puisse disposer d'un vaccin avant de nombreuses années encore [1].

\section{Les essais vaccinaux actuels}

Où en sommes-nous aujourd'hui ? En matière de vaccins vivants, on a totalement écarté l'idée d'utiliser des souches de virus atténué, par crainte qu'elles ne puissent s'avérer tumorigènes par intégration de leur LTR (long terminal repeat) à proximité d'un oncogène (LTR-activation). On craint trop aussi les possibilités de restauration accidentelle de la virulence par mutation réverse ou recombinaisons.

En revanche, des vaccins vivants recombinants, utilisant pour vecteurs le virus de la vaccine, ont été déjà testés chez les singes, le chimpanzé et même chez l'homme. Ont ainsi été testés des recombinants vaccine exprimant la glycoprotéine d'enveloppe gpl60 [2] (entière ou réduite à sa portion gpl20) ou les protéines virales Nef, Pol, Vif ou Gag. Dans l'ensemble, ces recombinants n'induisent qu'une assez modeste réponse humorale anti-HIV, mais une bonne réponse cellulaire. Ces réponses ne sont toutefois pas suffisantes pour protéger le chimpanzé contre une injection d'épreuve de virus virulent.

Les recombinants vaccine ne se prêtent pas non plus à la répétition des rappels, car une très forte immunité antivaccine s'établit après la deuxième injection ou scarification et empêche la multiplication du virus lors des injections ou scarifications suivantes. Pour contourner cette difficulté, Zagury [3] a eu l'idée de prélever les lymphocytes du sujet vacciné, de les infecter in vitro avec les recombinants vaccine voulus, puis de les fixer au formol avant de les réinjecter au donneur par voie intraveineuse. Ce rappel avec des cellules fixées s'est avéré très efficace mais la méthode est difficilement applicable à la vaccination sur le terrain.

En matière de vaccin inactivé, un vaccin constitué de virions entiers inactivés à la $\beta$-propiolactone et au formol et mélangés au muramyl dipeptide comme adjuvant a montré, chez le chimpanzé, un bon effet 
inducteur d'anticorps. La double vaccination par vaccin vivant recombinant suivi du vaccin inactivé n'a cependant pas permis de protéger l'animal contre une in jection d'épreuve de HIV, en dépit des forts taux d'anticorps anti-gag et anti-env et de la bonne réponse de prolifération cellulaire obtenus.

La situation paraît plus encourageante dans le cas du SIDA du singe, puisque l'équipe de Desrosiers a réussi à protéger deux singes Rhésus sur six contre une injection de SIV (simian immunodeficiency virus) virulent en utilisant un vaccin à base de virions inactivés par traitement avec un détergent [4]. Il est intéressant de noter qu'une première tentative de protection réalisée avec un vaccin anti-SIV inactivé au formol, une moindre charge antigénique, un nombre d'injections plus réduit, et des virions moins riches en glycoprotéine gpl20, s'était soldée par un échec. Il est donc vraisemblable que le mode de préparation du vaccin, sa concentration en antigène, la nature de l'adjuvant, l'espacement des injections, etc., jouent un rôle majeur dans ce domaine [1, 5].

En matière de vaccins contre des protéines virales isolées, enfin, on a testé chez le chimpanzé plusieurs préparations à base de glycoprotéines gpl20 ou gpl60 purifiées à partir du virus HIV ou produites par recombinaison génétique dans divers systèmes d'expression: cellule CHO (chinese hamster ovary cells), Escherichia coli, baculovirus, virus de la vaccine, etc. D'une manière générale, les glycoprotéines virales solubles ne sont pas de bons imm. urıogènes. C'est le fait d'être présentées à l'état particulaire à la surface du virion qui leur confère leur immunogénicité. La glycoprotéine gpl20 ne fait pas exception. Il n'est donc pas surprenant qu'en la seule présence du simple hydroxyde d'aluminium, qui est l'adjuvant classique des vaccins à usage humain, elle n'ait induit que des taux d'anticorps très faibles chez l'homme et chez le chimpanzé. Des réponses nettement plus encourageantes ont été obtenues chez le chimpanzé en utilisant des adjuvants à base de muramyl dipeptide ou en complexant l'antigène à des liposomes. L'emploi de complexes immunostimulants (ISCOMs: immunostimulating complexes) à $\mathrm{m} / \mathrm{s} \mathrm{n}^{\circ} 3$ vol. 5 , mars 89 base d'un dérivé de la saponine, semble donner des résultats plus prometteurs encore.

Cependant, aucun des chimpanzés immunisés jusqu'ici avec de la glycoprotéine gpl20 ne s'est avéré protégé contre l'injection d'épreuve de HIV. Plusieurs interprétations de ces échecs sont envisageables: l'insuffisance de la dose d'antigène et du nombre d'injections de vaccin, l'inefficacité de l'adjuvant, la nature même de la réponse immune aux divers épitotes de la glycoprotéine gpl20, ou encore le fait que la glycoprotéine d'enveloppe n'est pas le seul antigène à inclure dans un vaccin pour qu'il protège contre l'infection par le HIV. Il est évident que le nombre de paramètres intervenant ici est énorme et qu'un long travail sera nécessaire pour parvenir à les maîtriser.

\section{Un problème difficile...}

L'absence de précédent constitue de surcroît un lourd handicap dans la recherche d'un vaccin contre le SIDA. Certes, on sait vacciner efficacement contre deux rétrovirus agents de syndromes d'immunodéficience chez l'animal, le virus de Mason-Pfizer, rétrovirus de type $\mathrm{D}$, agent d'un des types de SIDA du macaque, et, avec des résultats moins probants, le FeLV, rétrovirus de type $\mathrm{C}$, agent de la leucémie féline et d'un des types de SIDA du chat. Mais on ne connaît aucun vaccin contre un rétrovirus appartenant comme le HIV ou le SIV à la famille des lentivirus. La mise au point d'un vaccin contre ces virus constitue à l'heure actuelle un redoutable défi lancé a u vaccinologue $[1,5,6]$.

Cela tient à plusieurs raisons : la variabilité antigénique du virus et sa faculté de se propager de cellule à cellule ou de se maintenir à l'état de provirus intégré dans l'ADN des cellules infectées lui permettent d'échapper aux défenses immunitaires (figure 1 , p. 154); les glycoprotéines de son enveloppe, gpl20 et gpl60, sont de mauvais immunogènes, et leur injection à l'animal n'induit que des titres médiocres d'anticorps neutralisants ; il règne une grande incertitude sur l'efficacité même de ces anticorps, de même que sur le rôle bénéfique des lymphocytes $T$ cytotoxiques dans la protection contre l'infection; l'ab- sence d'un bon modèle animal se fait cruellement sentir ; enfin, et ce n'est pas la moindre raison, toutes les tentatives réalisées jusqu'ici pour protéger des chimpanzés à l'aide de vaccins anti-HIV élaborés selon des recettes traditionnelles sont demeurées infructueuses. Plusieurs préparations vaccinales sont certes en cours d'essai clinique chez l'homme, mais elles n'ont fait preuve d'aucune efficacité dans les modèles animaux dont on dispose : ce sont au mieux des vaccins expérimentaux.

Il est intéressant d'analyser plus profondément ces différents aspects du problème.

Le premier obstacle sur la voie du vaccin est l'extraordinaire variabilité génétique dont fait preuve le HIV. Sa forte propension à muter, aussi bien en culture de cellules que dans l'organisme, engendre une variation antigénique considérable de ses protéines, notamment de ses protéines d'enveloppe. Or, c'est contre certains motifs exposés à la surface de la glycoprotéine gpl20, que sont dirigés les anticorps neutralisants. Le principal épitope reconnu par ces anticorps est formé d'une boucle de 30 acides aminés environ situés entre deux des cystéines d'une des régions hypervariables de la glycoprotéine gpl20 [7]. Ces acides aminés sont éminemment variables d'un isolat de virus à l'autre, sauf au sommet même de la boucle, constitué d'une proline entourée de deux glycines. C'est en changeant perpétuellement la séquence des acides aminés de cet épitope que le virus échappe à l'action des anticorps neutralisants élaborés par l'organisme pour l'éliminer [6].

L'hypervariabilité antigénique du virus HIV pose la question de savoir comment développer des vaccins susceptibles de protéger contre un très grand nombre d'isolats viraux à la fois. Ainsi, les sérums des animaux immunisés avec les vaccins prototypes à base de glycoprotéine gpl20, expérimentés jusqu'ici, montrent une activité neutralisante exclusivement vis-à-vis de l'isolat dont on a extrait la glycoprotéine gpl20 et non à l'égard des autres isolats. Cette étroite spécificité de la réponse expérimentale vis-à-vis de la protéine d'enveloppe du virus contraste avec la large spécificité de la réponse à l'infection naturelle. Les sérums de sidéens 
sont en effet capables de neutraliser un grand nombre d'isolats de HIV1 (ou de HIV-2) différents, et certains sérums anti-HIV-2 semblent même capables de neutraliser l'infectivité d'isolats du HIV-l (l'inverse n'a pas été observé). Il est probable que cela reflète l'émergence continuelle de nouveaux mutants au cours de la multiplication du virus dans l'organisme, mutants contre lesquels le système immunitaire réagit fidèlement en synthétisant les anticorps neutralisants appropriés. Dans le cas du vaccin, on ne sait pas comment faire pour induire d'emblée la panoplie d'anticorps neutralisants qui seraient nécessaires pour assurer la pleine protection du sujet vacciné contre tous les variants du virus.

Le deuxième obstacle au développement d'un vaccin contre le SIDA est l'extraordinaire capacité qu'a le virus de rester masqué dans certaines cellules de l'organisme pendant de longs mois, voire des années, ce qui lui permet d'échapper aux défenses immunitaires. Les porteurs asymptomatiques du virus développent pour la plupart de fortes réactions d'immunité, tant cellulaire qu'humorale, qui, dans le cas d'un autre virus, conduiraient rapidement à la guérison. Dans le cas du virus HIV, ces défenses parviennent bien à tenir le virus en échec relatif pendant quelque temps, mais elles ne permettent pas de l'éliminer. Cela est dû au fait que le virus peut échapper au système immunitaire en demeurant masqué à l'intérieur des cellules qu'il a infectées, vraisemblablement sous forme de provirus intégré dans l'ADN chromosomique et, dans le cas au moins des macrophages, sous forme de virions au repos dans des vacuoles intracytoplasmiques. Comme ni l'hypothétique cellule porteuse de provirus ni la cellule réservoir de virions n'expriment nécessairement des antigènes viraux à leur surface, elles échappent aux lymphocytes $\mathrm{T}$ cytotoxiques (CTL) et aux cellules tueuses (killer cells) chargées d'éliminer les cellules infectées.

Une fois entré en phase de multiplication active, le virus parvient à échapper encore aux défenses immunitaires et tout particulièrement aux anticorps neutralisants, en se propageant directement de cellule à cellule par le biais des fusions entre cellules saines $\mathrm{CD}^{+}$et cellules infectées exprimant la glycoprotéine gpl20 à leur surface: les cellules saines sont cooptées par les syncitia de cellules infectées et s'infectent à leur tour, sans qu'il soit nécessaire au virus de

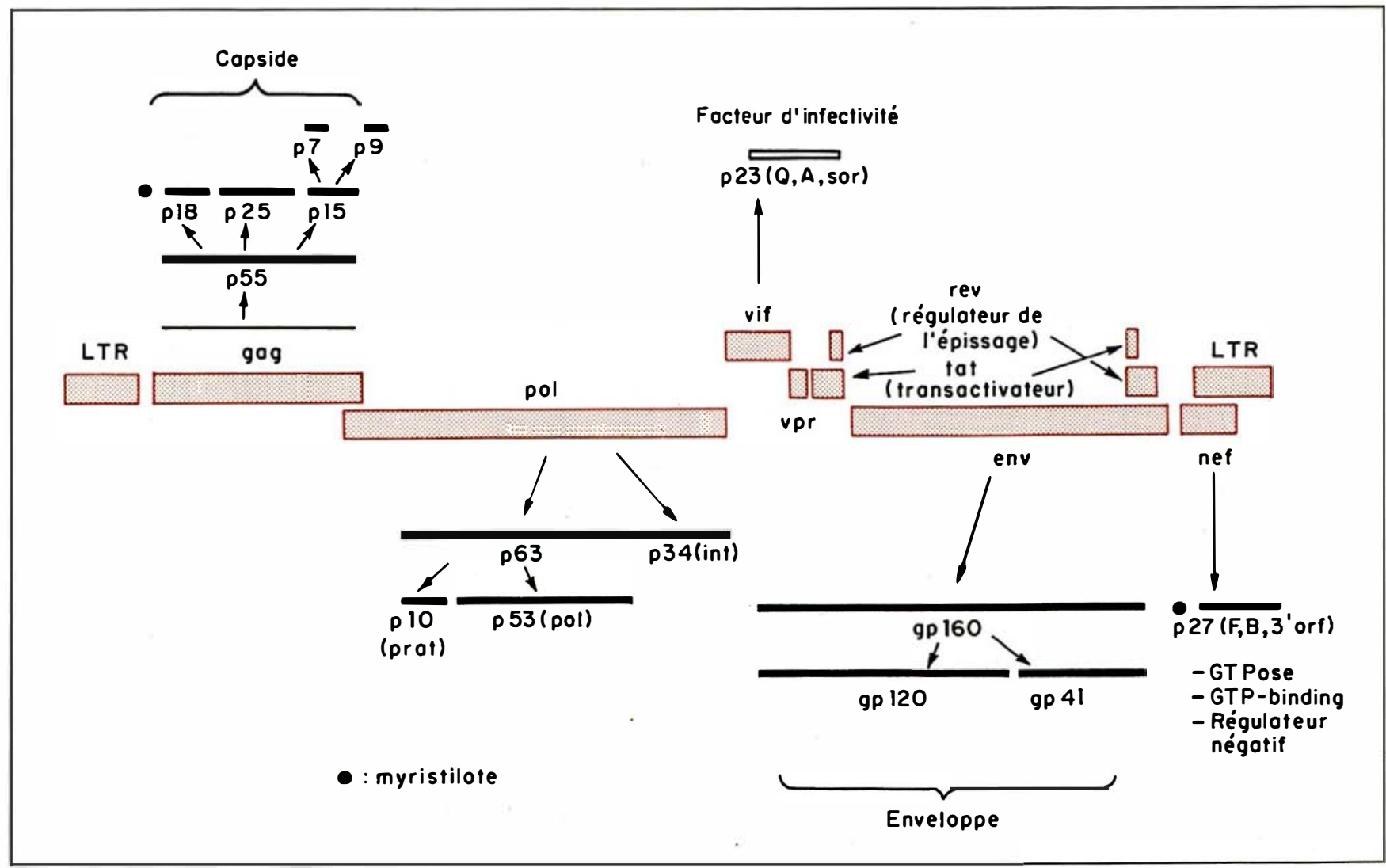

Figure 1. Schéma de l'organisation du génome du virus HIV-1 ot des cascades de synthèse et de protéolyse des produits de ses gènes. Prot = protéase ; pol = polymérase (transcriptase inverse); int = intégrase ; LTR = long terminal

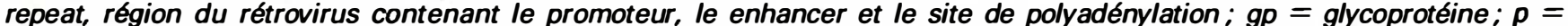
polypeptide ; $3^{\prime}$ orf $=3^{\prime}$ open reading frame, phase ouverte de lecture en $3^{\prime}$; GTP binding = liaison du GTP. Les indications entre parenthèse se réfèrent soit à la fonction des polypeptides (pol...), soit à leurs désignations alternatives (F, $B, 3^{\prime}$ orf). 


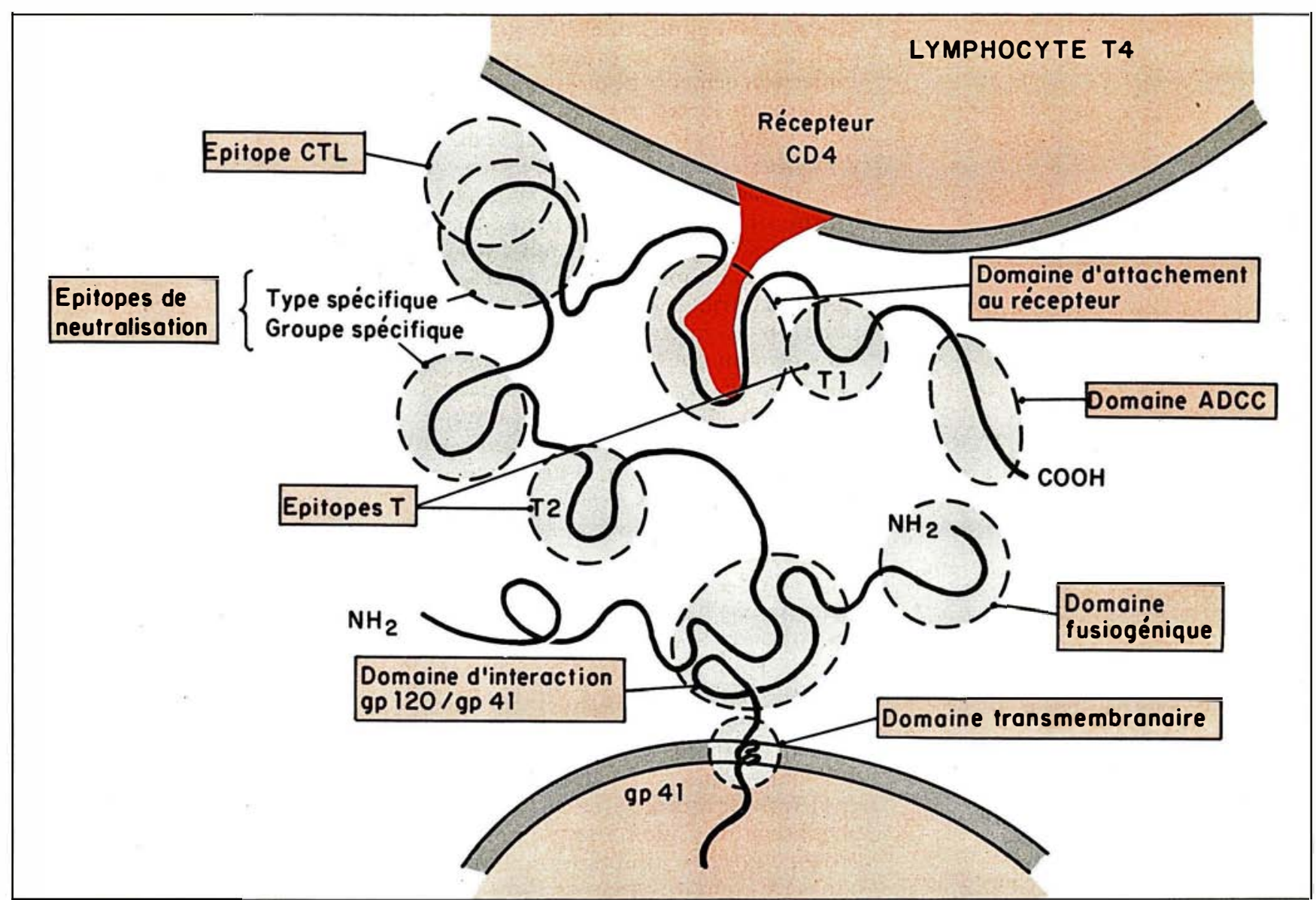

Figure 2. Représentation diagrammatique de la glycoprotéine gp120 ot de quelques-uns des domaines critiques. Epitopes $T$, épitope majeur de neutralisation, région impliquée dans l'attachement au récepteur CD4, domaine $A D C C$, domaine fusiogénique. ADCC = antibody dependent cellular cytotoxicity; CTL = cytotoxic $T$ lymphocyte.

passer par une étape extracellulaire. Enfin, le virus échappe aussi aux défenses de l'organisme en se réfugiant dans des compartiments tissulaires peu accessibles au système immunitaire, comme le système nerveux central.

\section{Rôle de l'immunité cellulaire}

L'efficacité d'un vaccin contre le SIDA implique donc en première analyse beaucoup plus que la seule propriété d'induire des anticorps neutralisants : elle nécessite aussi la capacité d'induire une immunité cellulaire à base de CTL ou de cellules tueuses armées d'anticorps (ADCC: antibody dependent cellular cytotoxicity)*.

L'infection par le HIV induit une $\mathrm{m} / \mathrm{s} n^{\circ} 3$ vol. 5 , mars 89 forte réponse immunitaire à médiation cellulaire chez l'homme et le chimpanzé, en particulier l'apparition de CTL de type $\mathrm{CD}^{+}$qui attaquent les cellules infectées et les détruisent. On a ainsi montré que les lymphocytes $\mathrm{T} 8$ isolés du poumon de malades atteints d'alvéolite lymphocytaire sont capables de tuer des cellules cibles exprimant à leur surface les antigènes env, gag ou nef du virus HIV-l en association avec la molécule de classe I du CMH (complexe majeur d'histocompatibilité) HLA-A2 [8]. On a, de même, montré l'existence de CTL dirigés contre les produits du gène pol [9].

* Voir $m / s$, Lexique immunologique, suppl. au $n^{\circ} 1$, vol. 5.
Ces CTL sont très efficaces in vitro [10]. In vivo, en revanche, chez le séropositif, ils sont inefficaces contre l'infection. Pour expliquer ce paradoxe, on a émis l'hypothèse que le virus pourrait se réfugier dans un compartiment de cellules souches peu différenciées, n'exprimant aucun antigène d'histocompatibilité à leur surface, et échappant de ce fait à toute reconnaissance par des CTL. Ces cellules serviraient de réservoir au virus. Si cette hypothèse s'avérait exacte, on ne pourrait se protéger de l'infection que si les anticorps et les CTL induits par le vaccin étaient suffisamment efficaces pour neutraliser le virus avant qu'il ait le temps de gagner le compartiment où il échappe à leur action, car une fois dans la place, le virus serait indélogeable. L'idée que le vaccin pourrait 


\section{RÉFÉRENCES}

1. Matthews T, Bolognesi D. Les vaccins contre le SIDA. Pour la Science 1988; 134 : 106-13.

2.Kieny MP, Lathe $\mathbf{R}$, Dott $\mathrm{K}$, et al. Improved antigenicity of the HIV env protein by cleavage site removal. Prot, Engineering $1988 ; 3$ : 219-25

3. Zagury D, Bernard J, Cheynier R, et al. A group-specific anamnestic reaction agains HIV induced by a candidate vaccine against AIDS. Nature 1988 ; 332 : 728-31.

4. Desrosiers RC. Simian immunodeficiency virus. In : Girard M, Valette L, eds. Retroviruses of Human AIDS and Related Animal Diseases. Lyon: Fondation Marcel-Mérieux, 1988: 190-3.

5. Fauci AS. Current issues in developing a strategy for dealing with the acquired immuno deficiency syndrome. Proc Natl Acad Sci USA 1988 ; 83 : 9278-83

6. Bolognesi D. Natural immunity ot HIV and its possible relationship to vaccine strategies. Microb Sciences 1988 ; 5 : 236-41.

7. Putney SD, Matthews T, Ribey WG, et al. HTLV-III/LAV neutralizing antibodies to an $E$. coli produced fragment of the virus envelope. Science 1986 ; 234 : 1392-5.

8. Plata F, Autran B, Pedroza-Martins L, et al. AIDS virus-specific cytotoxic T lymphocytes in lung disorders. Nature $1987 ; 328$ : 348-51.

9. Walker BD, Flexner C, Paraddis TJ, et al. HIV-1 reverse transciptase is a target for cytotoxic $\mathrm{T}$ lymphocytes in infected individuals. Science 1988 ; 240 : 64-6.

10. Zarling J, Eichberg JW, Moran PA, et al. Proliferative cytotoxic $T$ cells to AIDS virus glycoproteins in immunized chimpanzees. J Immunol 1987 ; 139 : 988-90

11. Robinson WE, Montefiori DC, Mitchell WM. Will antibody-dependent enhancement of HIV-1 infection be a problem with AIDS encore néanmoins protéger contre la maladie sans avoir protégé contre l'infection demeure pour l'instant du domaine de la spéculation.

Cependant, les lymphocytes T8 cytotoxiques induits en réponse à l'infection par le HIV ne jouent pas uniquement un rôle bénéfique: au contraire, c'est à leur action cytolytique sur les macrophages infectés que l'on attribue, par exemple, l'apparition de zones inflammatoires dans les poumons ou le cerveau des sujets infectés. Les CTL contribuent, de surcroît, directement à amplifier les symptômes d'immunosuppression en détruisant les lymphocytes $\mathrm{T} 4$, les lymphocytes $\mathrm{B}$ et les macrophages portant des antigènes du virus HIV à leur surface. Les CTL spécifiques de la glycoprotéine gpl20 provoquent, par exemple, la lyse massive des lymphocytes T4, car ces derniers fixent passivement la glycoprotéine de l'enveloppe virale sur leurs récepteurs CD4. La glycoprotéine gpl20 (figure 2, p. 155) n'est que très mal arrimée à la glycoprotéine gp4l qui est censée la retenir sur la membrane. Des molécules de glycoprotéine gpl20 «soluble» sont donc constamment relarguées dans le milieu extracellulaire par les cellules infectées ou à partir des virions. Elles y jouent le rôle d'un double leurre: vis-à-vis des anticorps neutralisants, qu'elles piègent sous forme de complexes antigène-anticorps ; et vis-àvis des CTL, dont elles dirigent l'attaque vers les lymphocytes T4 en se fixant avec une grande affinité sur les récepteurs $\mathrm{CD} 4$. On a récemment émis l'hypothèse que les cellules T4, ayant fixé puis internalisé la glycoprotéine gpl20, seraient reconnues et détruites par les CTL dès lors qu'elles en présenteraient des fragments à leur surface en association avec les molécules de HLA de classe II.

\section{Anticorps facilitants et anticorps neutralisants}

La nécessité, pour un vaccin, d'induire des CTL spécifiques de la glycoprotéine gpl20 pourrait donc bien être remise en cause par ces observations. Il faut noter, cependant, que la situation n'est pas claire et que le rôle des CTL pourrait être en fait d'autant plus important que l'on a émis des doutes sur l'efficacité des anticorps neutralisants dans la protection contre le HIV. Des anticorps humains, parfaitement capables de neutraliser l'infectivité d'un isolat de HIV-1 pour des cellules de la lignée lymphocytaire $T 4$, s'avèrent en effet incapables de bloquer l'infectivité du même isolat de virus pour des cellules de la lignée monocytaire. Par ailleurs, l'administration au chimpanzé d'anticorps neutralisants humains, à dose massive, ne s'avère pas capable de protéger passivement l'animal contre une dose d'épreuve de HIV-l, qu'ils neutralisent pourtant parfaitement en culture de cellules. Bien au contraire, les animaux immunisés passivement semblent devenir virémiques plus rapidement que les animaux témoins. On a tiré de ces diverses observations la conclusion que les anticorps neutralisants, loin d'être bénéfiques, pourraient se révéler à tout le moins facilitants.

Le phénomène de facilitation (immune enhancement) a été initialement décrit dans lecas de l'infection par le virus de la dengue par Halstead en 1977 : l'addition d'anticorps antidengue à concentration subneutralisante favorise l'infection des monocytes humains en culture. Cela tient au fait que les monocytes/macrophages peuvent fixer la portion Fc des immunoglobulines par leurs récepteurs Fc $\gamma$. Ils peuvent donc s'infecter soit directement par le virus, soit indirectement lors de l'endocytose des complexes virus-anticorps au niveau du récepteur $\mathrm{F}_{\mathrm{c}} \gamma$. Cet effet est décuplé par l'addition d'interféron $\gamma$, dont on sait qu'il active l'expression du gène du récepteur $F_{c} \gamma$. Le phénomène de facilitation rappelle à l'évidence le phénomène d'opsonisation * bien connu en bactériologie.

Dans le cas de la dengue clinique, c'est à un phénomène de facilitation qu'on attribue l'origine des cas graves, très souvent mortels, de dengue hémorragique ou de dengue avec syndrome de choc (dengue haemorrhagic fever-dengue shock syndrome: DHF-DSS). La plupart des cas de

\footnotetext{
* Opsonisation: facilitation de la phagocytose. Une opsonine est un anticorps dont l'union avec l'antigène correspondant facilite la capture par les cellules phagocytaires. Le facteur C3 du complément a également un pouvoir opsonisant.
} 
DHF-DSS s'observent à l'occasion d'un deuxième épisode de dengue provoqué par un virus de sérotype différent de celui responsable du premier. Les anticorps qui persistent de la première infection ne sont pas neutralisants vis-à-vis du deuxième virus, mais ils forment avec lui des complexes virus-anticorps dont l'endocytose entraîne et facilite l'infection du macrophage. On les qualifie pour cette raison d' «an ticorps facilitants ».

Dans le cas du virus HIV, des anticorps facilitants ont pu être mis en évidence dans certains sérums de sidéens. Ils y coexisteraient avec les anticorps neutralisants, qu'ils parviennent même à masquer [11]. Cette observation nécessite cependant d'être confirmée, car l'existence d'un phénomène de facilitation n'a été démontrée de façon formelle in vivo ni chez le chimpanzé dans le cas du HIV ni chez le macaque dans le cas du SIV. Il est toutefois permis de penser que des anticorps anti-gpl20 non neutralisants pourraient faciliter l'infection des macrophages et jouer un rôle facilitant. Si tel était le cas, on devrait s'abstenir d'utiliser les glycoprotéines (gpl20 ou gpl60) comme immunogènes dans les vaccins, ou du moins les déléter au préalable de leurs épitopes de facilitation éventuels.

L'idéal sera peut-être, dans le futur, de n'utiliser que des fragments de glycoprotéines gpl 20 ou gpl 60 contenant les seuls épitopes inducteurs d'anticorps neutralisants, ou des oligopeptides de synthèse de séquence correspondant à l a séquence de ces épitopes, ou encore des particules ou des antigènes chimères dans lesquels on aura inséré par recombinaison génétique la séquence des épitopes en question. Plusieurs démarches expérimentales ont été entreprises dans ce sens tant avec le virus de l'hépatite $B$ (particules d'antigène $\mathrm{HBs}$ ou $\mathrm{HBc}$ hybrides comportant des épitopes de la glycoprotéine gpl60) qu'avec le poliovirus (souche Sabin chimère exposant un épitope de la glycoprotéine gpl 60 à la surface de sa capside) ou avec des bactéries (entérobactéries exprimant à leur surface des épitopes de la gpl60 insérés dans la protéine membranaire LamB).

Ces nouvelles approches expérimentales sont d'autant plus intéressantes

$\mathrm{m} / \mathrm{s} n^{\circ} 3$ vol. 5 , mars 89 qu'il ne s'est pas avéré, jusqu'ici, possible d'induire des taux élevés d'anticorps neutralisants chez les animaux d'expérience, notamment le chimpanzé, ni par injection de préparations à base de glycoprotéines gpl 20 ou gpl 60 ou de virions entiers inactivés ni par injection de virus de la vaccine recombinant exprimant la glycoprotéine gp 160. L'expérience nous a montré que certaines préparations de la glycoprotéine gpl 60 mélangées au muramyl dipeptide comme adjuvant pouvaient induire chez le chimpanzé des taux d'anticorps supérieurs au 1/100 000 (mesurés par Elisa) sans s'accompagner pour autant de titres d'anticorps neutralisant significatifs. Tout se passe comme si les épitopes de neutralisation passaient inaperçus du système immunitaire, ou comme s'il se mettait en place un phénomène encore hypothétique de suppression spécifique de ces épitopes.

Mais les anticorps neutralisants sontils nécessaires ? sont-ils effectivement protecteurs in vivo? La question a rebondi récemment avec l'observation que, selon leur nature, certains anticorps pouvaient être neutralisants en culture de cellules sans l'être dans l'organisme, alors que d'autres au contraire manifestaient une efficacité identique dans les deux circonstances. Ainsi, alors qu'un échantillon de HIV-1, mélangé avec un sérum de sidéen dans des proportions telles qu'il avait perdu totalement son infectivité pour des cellules T4 en culture, demeurait infectieux pour le chimpanzé, un échantillon analogue mélangé avec le sérum d'un chimpanzé ayant développé des anticorps neutralisants à la suite de son infection par le HIV perdait au contraire totalement son infectivité, aussi bien pour l'animal que dans le système de culture de cellules. Les anticorps neutralisants du chimpanzé semblent donc bien avoir été capables de protéger l'animal contre l'infection, mais non les anticorps d'origine humaine. Le titre d'anticorps neutralisants du sérum de chimpanzé utilisé dans cette expérience, 1/640, pourrait représenter dans l'avenir le seuil qu'il serait désirable d'atteindre après vaccination pour conférer un début de protection contre l'infection.

Cependant, la notion que les anticorps neutralisants sont indispensa- bles pour la protection reste débattue. La théorie selon laquelle les anticorps anti-gpl 20 provoquent un phénomène de facilitation conduit même à prédire qu'il ne faudrait développer de vaccins qu'à base des protéines virales internes (Gag et Pol) ou non structurales. D'ailleurs, les séropositifs ont tendance à perdre progressivement leur anticorps anti-pl 8 et anti-p25 gag en même temps qu'apparaissent puis s'amplifient les symptômes de leur maladie. Cette observation a conduit Salk à prédire qu'on pourrait arrêter, sinon même renverser, le cours de la maladie, en stimulant le système immunitaire avec des préparations des seuls antigènes p25 et pl8 gag. Plusieurs équipes californiennes s'employent à vérifier expérimentalement cette hypothèse. $\mathrm{Si}$ cette démarche était couronnée de succès chezles séropositifs, il conviendrait de déterminer si elle peut s'appliquer aussi au cas de la vaccination préventive chez les séronégatifs. Le même vaccin serait alors utilisable commeagent immunothérapeutique et comme agent prophylactique.

En l'absence de bons modèles animaux, on ne pourra réellement tester l'efficacité des vaccins anti-SIDA et les différentes théories qui s'affrontent quant à leur conception que chez l'homme [1]. Les études cliniques entreprises à l'heure actuelle, tant en France ou au Zaïre qu'aux ÉtatsUnis, en Angleterre ou en Suisse, ne sont que des études de phase I réalisées avec des préparations dont l'efficacité n'a pas été démontrée dans le seul système de validation dont on dispose, celui du chimpanzé. Il faut espérer que l'on parviendra à obtenir dans le futur des vaccins doués d'efficacité chez le chimpanzé, dont on pourra alors sans réserve tester l'efficacité chez l'homme dans des études cliniques mettant en jeu plusieurs centaines de volontaires, ce qui ne manquera sans doute pas de poser alors d'autres problèmes. Mais ceci est une autre histoire

\section{TIRÉS A PART}

M. Girard. 


\section{Summary}

AIDS vaccines: hopes and realities

The possibility of developing an AIDS vaccine seems to still be quite remote due to the constant antigenic variation of the virus outer envelope glycoproteins gpl60/gpl20, in which is located the major neutralization epitope, and due to the fact that the virus can escape immune defense mechanisms by remaining hidden for long periods of time inside infected cells, probably under the form of an integrated provirus, or at least in the case of macrophages, as inactive virions inside cytoplasmic vacuoles. The virus can also dodge the immune response by passing directly from infected to uninfected cell withoug having to go through an extracellular phase, and by taking refuge in the central nervous system. All attempts at protecting chimpanzees against infection with HIV-1 by immunization with a variety of experimental vaccines have failed so far : vaccinia virus recombinants expressing gpl20 or gpl60env and p25gag ; formalin and betapropiolactone inactivated whole HIV virions : and purified gpl 20 or gpl60 injected in the presence of alum or other adjuvants. There is therefore no criterion today from which to judge whether neutralizing antibodies, or cytotoxic T-cells, or both, are required for protection, nor what is the minimal level of each required. Neutralizing antibodies have been accused of playing a role in facilitation, allowing easier infection of the macrophage. Cytotoxic T-cells are responsible for some of the observed lung and brain pathology in AIDS patients, and do actually contribute to the loss of $\mathrm{T} 4$ lymphocytes by attacking gpl20-presenting $\mathrm{CD}^{+}$cells. Contradictory theories have been proposed to account for these phenomena, but none has been really tested yet. A few glimmers of hope have however been sparked recently with the demonstration that partial protection against the Simian immunodeficiency virus, SIV, could be conferred to Rhesus macaques by vaccination with an inactivated virus vaccine, and by showing that neutralizing antibodies from a chimpanzee were able to protect another chimpanzee against infection with HIV-l. We still are a long way from a vaccine which would show efficacy in a chimpanzee protection test and could be safely tested for efficacy in a large number of humans at risks, but these observations foster the hope that we will reach that stage eventually. 